\title{
Erratum: QED with a spherical mirror [Phys. Rev. A 82, 063812 (2010)]
}

\author{
G. Hétet, L. Slodička, A. Glätzle, M. Hennrich, and R. Blatt
}

(Received 28 July 2020; published 31 August 2020)

DOI: 10.1103/PhysRevA.102.029902

Prefactors appear to have been missing in the formulas of the modified spontaneous decay and levels shift of an atom close to the focus of a spherical mirror presented in our paper. Here, we give the correct formulas together with the corrected plots showing the evolution of the decay and shifts as a function of numerical aperture and distance from the spherical mirror.

Decay rate. In the original paper, a factor of $\pi$ in Eq. (4) and a factor of 2 were missing when taking the continuum limit in Eq. (20). Including these factors and the two photon polarizations, we recover the free space decay rate [1],

$$
\gamma_{\mathrm{FS}}=\frac{4 d^{2} \omega_{0}^{3}}{3 \hbar c^{3}}
$$

where $d$ is the atomic dipole constant and $\omega_{0}$ is the atomic transition frequency. The goal of the paper was to calculate the modified decay and level shifts for a single dipole. The typically richer multilevel structure of real atoms [2] implies isotropic field emission. The factor of $3 / 2$ should, thus, not appear in Eq. (58) of our paper. Equation (58) of the text should, instead, read

$$
\begin{aligned}
\left|\phi_{\vec{k}}(\vec{r})\right|^{2}= & \frac{k^{2}}{\Lambda} \int_{2 \pi} \frac{d \vec{\Omega}}{4 \pi}\left(1-\left|\frac{\vec{d} \cdot \vec{\Omega}}{d}\right|^{2}\right) \\
& \times\{1-\rho \cos [2(k R+\vec{k} \cdot \vec{r})]\} .
\end{aligned}
$$

It follows that,

$$
\gamma(\vec{r})=\frac{4 d^{2} \omega_{0} \Lambda}{\hbar c}\left|\phi_{\vec{k}_{0}}(\vec{r})\right|^{2} .
$$

The change in the decay rate as a function of the mirror numerical aperture is plotted in Fig. 1 considering a dipole that is perpendicular to the mirror axis. Notably, the change in the decay rate at a numerical aperture $(\mathrm{NA})=0.4$ is now two times smaller, which is closer to the experimentally observed values [2].

The change in the decay rate is also plotted in Fig. 2 as a function of its distance from the focus in the case of a fullhemispherical mirror.

Levels shifts. Similarly, the Lamb-shift formula found in Eq. (56) should be corrected. The ground- and excited-state shifts read

$$
\Delta_{e, g}(\vec{r})=\frac{2 d^{2} \omega_{0}^{2} \Lambda}{\pi \hbar c^{2}} P \int_{0}^{+\infty} \frac{d k}{k\left(k \pm k_{0}\right)}\left|\phi_{\vec{k}}(\vec{r})\right|^{2} .
$$

In the one-dimensional case considered in the original paper, the Lamb shift at Eq. (10) should, thus, be

$$
\Delta_{e}(0)=-\frac{\gamma_{\mathrm{FS}}}{2} \sin \left(2 k_{0} R\right),
$$

in agreement with Ref. [3]. In the case of a hemispherical mirror, the normalized Lamb shift is

$$
\begin{aligned}
\bar{\Delta}_{g, e}(\vec{r})= & -\frac{3}{2} \int_{2 \pi} \frac{d \vec{\Omega}}{4 \pi}\left(1-\left|\frac{\vec{d} \cdot \vec{\Omega}}{d}\right|^{2}\right) \rho \\
& \times \sin \left[2\left(k_{0} R+\vec{k}_{0} \cdot \vec{r}\right)\right] .
\end{aligned}
$$

Figure 3 shows the level shifts in the ground (trace iii) and excited state (trace ii) as a function of the mirror distance from the atom. Note that the level shifts oscillate between $-\gamma_{\mathrm{FS}} / 2$ and $\gamma_{\mathrm{FS}} / 2$ with a peak-to-peak amplitude $\gamma_{\mathrm{FS}}$.

In conclusion, we provided corrections for the formulas for the decay and shifts of the electronic state of a single atom at the focus of a hemispherical mirror given in our paper. Although the newly predicted changes in the decay and shifts are smaller, the main conclusions of the paper, namely, that the spontaneous decay rate and level shifts of an atom can

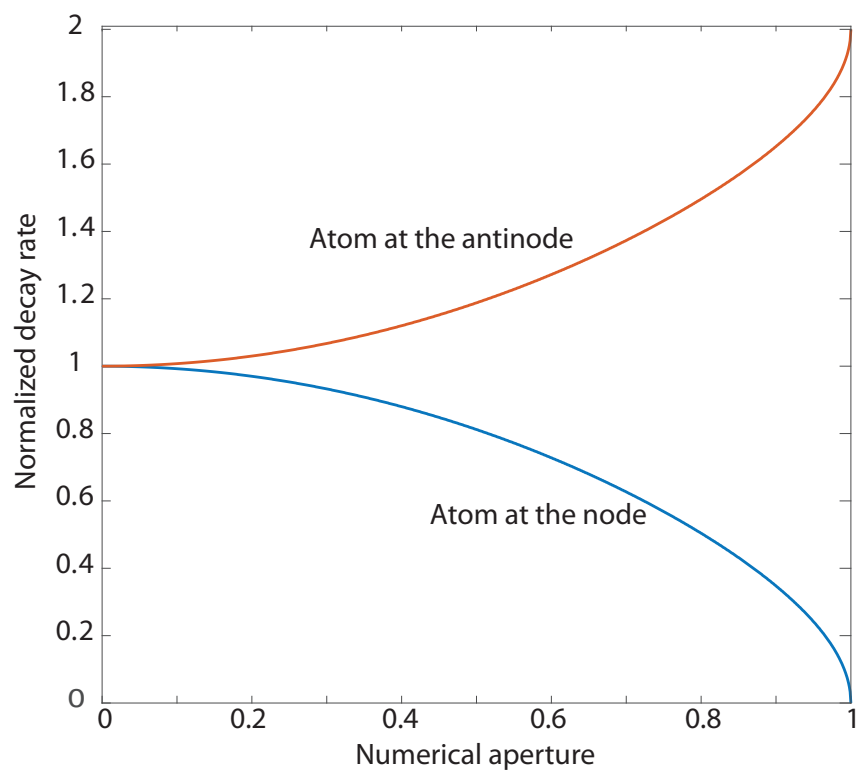

FIG. 1. Spontaneous decay rate as a function of the spherical mirror numerical aperture for an atom at the mirror focus, at the antinode, or at the node of the standing wave. 


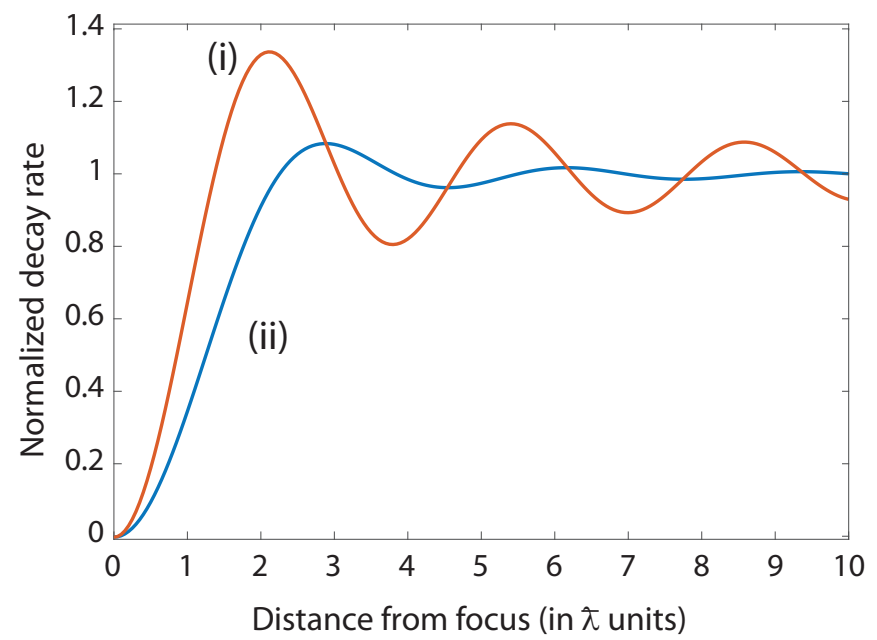

FIG. 2. Spontaneous decay rate of an atom as a function of its distance from the focus in the case of a full-hemispherical mirror. Here, the mirror is positioned such that a node of the standing wave is at the focus. Trace (i) corresponds to a scenario where the atom is displaced along the mirror axis. Trace (ii) is when the atom is displaced perpendicular to the mirror axis.

be suppressed by a hemispherical mirror and the $\propto 1 /(k R)^{2}$ Casimir-Polder scaling remain unchanged.

We would like to thank G. Cerchiari, E. Lassale, and D. Wilkowski for fruitful discussions. This research has been

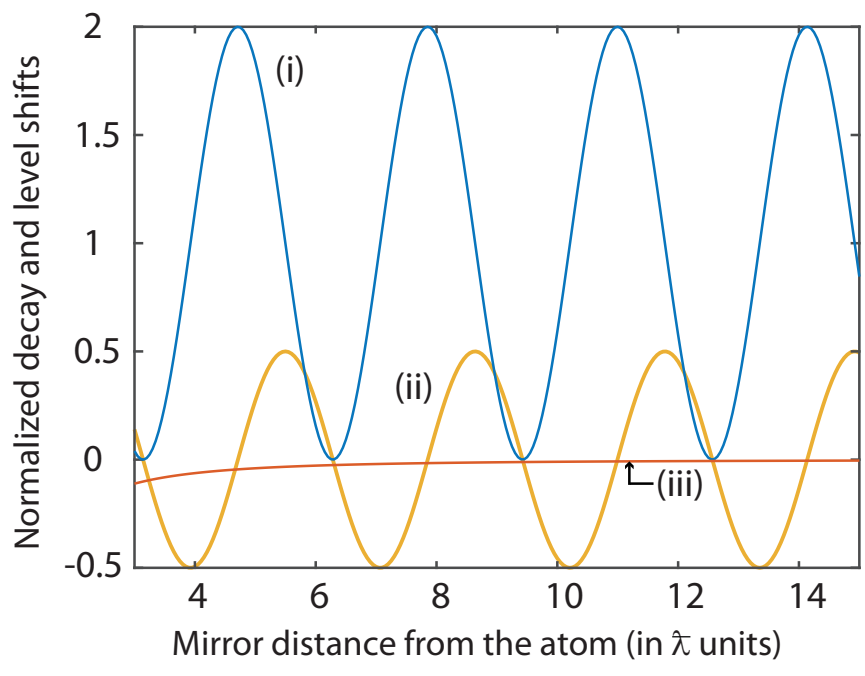

FIG. 3. Normalized spontaneous decay rate (i) and excited- (ii) and ground-state (iii) shifts of a dipole as a function of the mirroratom distance in the case of a full-hemispherical mirror. The atom is at the mirror focus.

partially funded by the French National Research Agency (ANR) through the T-ERC Project QUOVADIS. This work was supported by the European Commission through the Marie Skłodowska-Curie Action Grant No. 801110 (Erwin Schrödinger Quantum Fellowship Programme).
[1] The Quantum Vacuum, edited by P. W. Milonni (Academic, San Diego, 1994), pp. 115-153.

[2] J. Eschner, C. Raab, F. Schmidt-Kaler, and R. Blatt, Nature (London) 413, 495 (2001).
[3] U. Dorner and P. Zoller, Phys. Rev. A 66, 023816 (2002). 